\title{
Atomic force microscopy measurements of topography and friction on dotriacontane films adsorbed on $\mathrm{a} \mathrm{SiO}_{2}$ surface
}

\author{
S. Trogisch, M. J. Simpson, and H. Taub ${ }^{\text {a) }}$ \\ Department of Physics and Astronomy and University of Missouri Research Reactor, \\ University of Missouri-Columbia, Columbia, Missouri 65211 \\ U. G. Volkmann and M. Pino \\ Facultad de Física, Pontificia Universidad Católica de Chile, Santiago 22, Chile \\ F. Y. Hansen \\ Department of Chemistry, Technical University of Denmark, IK 207 DTU, DK-2800 Lyngby, Denmark
}

(Received 21 February 2005; accepted 17 August 2005; published online 18 October 2005)

\begin{abstract}
We report comprehensive atomic force microscopy (AFM) measurements at room temperature of the nanoscale topography and lateral friction on the surface of thin solid films of an intermediate-length normal alkane, dotriacontane $\left(n-\mathrm{C}_{32} \mathrm{H}_{66}\right)$, adsorbed onto a $\mathrm{SiO}_{2}$ surface. Our topographic and frictional images, recorded simultaneously in the contact mode, reveal a multilayer structure in which one to two layers of molecules adsorb adjacent to the $\mathrm{SiO}_{2}$ surface oriented with their long axis parallel to the interface followed by partial layers of molecules oriented perpendicular to the surface. The thicknesses of the parallel and perpendicular layers that we measured with the AFM agree with those inferred from previous x-ray specular reflectivity measurements on similarly prepared samples. We also observe bulk dotriacontane particles and, in contrast with our previous measurements, are able to determine their location. Above a minimum size, the bulk particles are separated from islands of perpendicularly oriented molecules by regions of exposed parallel layers that most likely extend underneath the particles. We find that the lateral friction is sensitive to the molecular orientation in the underlying crystalline film and can be used effectively with topographic measurements to resolve uncertainties in the film structure. We measure the same lateral friction on top of the bulk particles as on the perpendicular layers, a value that is about 2.5 times smaller than on a parallel layer. Scans on top of parallel layers indicate a constant height but reveal domains having different sublevels of friction. We explain this by the domains having different azimuthal orientations of the molecules. (C) 2005 American Institute of Physics.
\end{abstract}

[DOI: $10.1063 / 1.2060707]$

\section{INTRODUCTION}

As alkane $\left(\mathrm{C}_{n} \mathrm{H}_{2 n+2}\right)$ molecules are the principal constituents of commercial lubricants, it is of interest to characterize the structure of both liquid and solid alkane films at the molecular level and to correlate their structures with the lateral frictional force that they exert on a nanoscale object. Atomic force microscopy (AFM) has developed rapidly in recent years for this purpose. ${ }^{1}$ For example, AFM measurements on polydiacetylene monolayers have revealed a large friction anisotropy, which can be correlated with the molecular orientation and translational ordering in the film., ${ }^{2,3}$ However, to our knowledge, such studies have not been conducted previously with alkanes or with other organic molecules for which the solid film exhibits more than one crystallographic structure. We shall see that, when deposited from solution onto a $\mathrm{SiO}_{2}$ surface, films of intermediatelength alkanes possess two crystalline phases in which the long axis of the molecules is oriented either parallel or perpendicular to the surface, respectively. In addition, at higher coverages these quasi-two-dimensional phases coexist with

${ }^{a)}$ Electronic mail: taubh@missouri.edu preferentially oriented bulk particles. This film polymorphism produces a rich topography with which to correlate both transnational and molecular orientational orders with lateral friction.

Studies of the wetting of intermediate-length alkanes $(15<\mathrm{n}<50)$ to solid surfaces have been stimulated by the discovery over ten years ago of an intriguing surface freezing effect at the bulk alkane fluid/air interface. In the surface freezing effect, a solid molecular layer immediately adjacent to the air interface persists a few degrees above the bulk melting point. ${ }^{4}$ The molecules in this monolayer phase are predominantly in their trans configuration and form a hexagonal lattice with their long-axis oriented perpendicular to the interface. This behavior contrasts with that of surface melting in which an interfacial layer melts at a temperature below the bulk melting point.

Riegler and co-workers ${ }^{5-8}$ have proposed that a similar surface freezing effect can occur with intermediate-length alkane molecules if air is replaced by a solid substrate $\left(\mathrm{SiO}_{2}\right)$. In their model of a thick alkane film, a crystalline monolayer of perpendicularly oriented alkane molecules adsorbs immediately adjacent to the $\mathrm{SiO}_{2}$ surface. Above this perpendicular monolayer, solid bulk alkane particles nucleate 
and melt into droplets upon heating to the bulk melting point. Complete wetting of the alkane film occurs a few degrees above the bulk melting point.

Volkmann et $a l^{9}{ }^{9}$ have used high-resolution ellipsometry to investigate the interfacial structure of an intermediatelength alkane film, dotriacontane $\left(n-\mathrm{C}_{32} \mathrm{H}_{66}\right.$ or $\left.\mathrm{C} 32\right)$, deposited from a solution with heptane onto $\mathrm{SiO}_{2}$-coated $\mathrm{Si}(100)$ single-crystal substrates. The growth mode that they inferred differs in an essential way from that proposed earlier ${ }^{5,6}$ by including molecular layers adjacent to the $\mathrm{SiO}_{2}$ surface in which the long axis of the $\mathrm{C} 32$ molecules is parallel to the interface.

Synchrotron x-ray specular reflectivity measurements on similarly prepared $\mathrm{C} 32$ films deposited onto $\mathrm{SiO}_{2}$-coated $\mathrm{Si}(100)$ substrates ${ }^{10}$ have supported the existence of a parallel film phase immediately adjacent to the solid interface followed by adsorption of a perpendicular monolayer. At higher coverages and below the bulk C32 melting point, preferentially oriented bulk crystallites were observed having either the previously reported monoclinic structure ${ }^{11}$ or a new, presumably surface stabilized, orthorhombic structure.

Although these x-ray specular reflectivity measurements on $\mathrm{C} 32$ films grown from solution on $\mathrm{SiO}_{2}$ (Ref. 10) were consistent with one to two layers of parallel molecules underneath a perpendicular monolayer as inferred by highresolution ellipsometry, ${ }^{9}$ there have not yet been direct measurements of the morphology and topography of these samples. It would be desirable to determine the lateral extent of both the parallel and perpendicular layers as well as confirm their stacking sequence. While ellipsometric and x-ray techniques average over a sample area greater than $\sim 1 \mathrm{~mm}^{2}$, the smaller sampling area achievable with AFM $\left(<10^{-2} \mathrm{~mm}^{2}\right)$ allows measurement of the lateral dimensions of the molecular layers and bulk particles. AFM can also be used to determine the location of the bulk particles, i.e., where they reside with respect to the parallel and perpendicular layers of the film.

There have not been any previous reports of AFM measurements on $\mathrm{C} 32$ adsorbed on $\mathrm{SiO}_{2}$. However, AFM topographic images using the tapping mode have been obtained for $\mathrm{C} 30$ adsorbed on $\mathrm{SiO}_{2}$, ${ }^{6,7}$ which is believed to have a similar growth mode as $\mathrm{C} 32$. Some topographic images of $\mathrm{C} 30$ at submonolayer coverage show fractal structures ${ }^{6}$ whereas others show a nearly complete alkane monolayer with step heights in the range of $2.0-2.3 \mathrm{~nm}^{6}$ These step heights are less than the length of a fully extended C30 molecule $(\sim 41 \AA)$ and cannot be reconciled easily with the perpendicular monolayer thickness that the authors inferred earlier by $\mathrm{x}$-ray specular reflectivity measurements. ${ }^{5-7}$

In this paper, we report AFM measurements that confirm the existence of one to two parallel layers underneath a perpendicular layer in solid C32 films deposited from solution onto a $\mathrm{SiO}_{2}$ surface. The layer thicknesses agree well with those inferred in our previous x-ray specular reflectivity measurements ${ }^{9}$ and, in addition, we can determine the lateral extent of these layers. In some cases, we find multiple perpendicular layers to be present. We are also able to determine the size and location of coexisting bulk C32 particles as well as investigate their structure and morphology. Finally, we compare the lateral frictional forces exerted on the AFM tip by the surfaces of the parallel and perpendicular layers with that exerted by the surface of the bulk particles.

\section{EXPERIMENT}

The AFM measurements reported here were conducted on C32 films deposited from solution onto $\mathrm{Si}(100)$ substrates of an area of $12 \times 12 \mathrm{~mm}^{2}$ coated with their native oxide. The $\mathrm{SiO}_{2}$ coating had a thickness in the range of $12-25 \AA$ as determined by modeling of specular reflectivity scans of similarly prepared samples ${ }^{10}$ and was assumed to be amorphous. These substrates were cleaned by two different methods. The first method, which we refer to as "UV cleaning," consisted of washing the substrate with heptane $\left(\mathrm{C}_{7} \mathrm{H}_{16}\right)$, drying it in a nitrogen environment, and finally exposing it to UV light. We also performed AFM measurements on samples cleaned in this way but, as in the ellipsometry experiments, ${ }^{9}$ without exposure to UV light. However, we believe that these substrates retained more organic impurities; and, since they did not reveal any qualitatively new features, we do not present results on these samples here. In the second or "acid cleaning" method, the substrate was treated in a $\mathrm{H}_{2} \mathrm{O}_{2}+$ sulfuric acid mixture to remove organic contamination as used previously. ${ }^{12}$

The clean substrates were dip coated in a C32/heptane solution following the procedures used in previous ellipsometry ${ }^{9}$ and x-ray ${ }^{10}$ experiments. After dip coating and before the AFM experiments, we measured the thickness of the C32 films by high-resolution ellipsometry. ${ }^{9}$ Two different solution concentrations were used: a solution of $11.55 \mathrm{mg}$ of C32 dissolved in $20 \mathrm{ml}$ of heptane yielded C32 films of thickness $\sim 65 \AA$; a higher concentration solution $(18.97 \mathrm{mg}$ of C32 in $20 \mathrm{ml}$ of heptane) gave samples that were $\sim 95 \AA$ thick. We also investigated samples with a higher C32 coverage $(\sim 140 \AA$ thick) on UV-cleaned substrates, but these did not reveal any additional features.

Our AFM measurements were performed with a Nanoscope IIIa (formerly Digital Instruments, Inc. now Veeco Instruments, Inc.) operating in the contact mode in order to measure topography and friction simultaneously. We note that using the tapping mode on soft matter at fixed driving frequency has been shown to yield false step heights between layers of different materials. ${ }^{13,14}$ To avoid these artifacts and to minimize the damage done to the soft organic film, we used contact-mode cantilevers with small spring constants $(0.01-0.03 \mathrm{~N} / \mathrm{m})$. Their tips were pyramidal in shape with a radius of curvature in the range of $10-40 \mathrm{~nm}$.

The lateral frictional force causes torsion of the microscope tip about an axis oriented perpendicular to the scan direction and parallel to the surface. It is this torsional angle that is recorded as the friction signal. Since the lateral frictional force depends on the magnitude of the normal force exerted by the cantilever, we first determined the normal force by recording a force versus a distance curve. In this way, we established that the variation in the lateral frictional force was not caused by changes in the normal force. The magnitude of the normal force $(<10 \mathrm{nN})$ was also used to test for tip damage and/or contamination of the tip. 
(a)

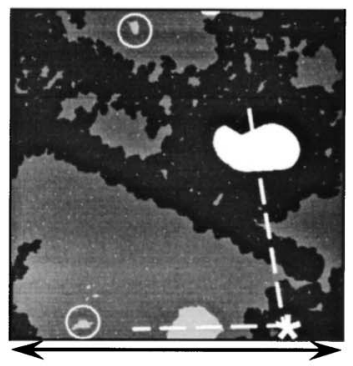

(b)

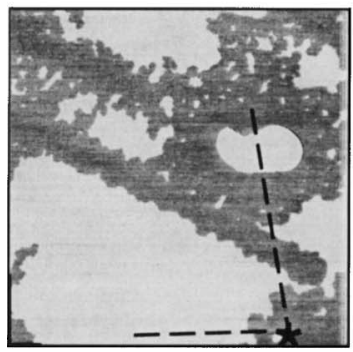

(c)

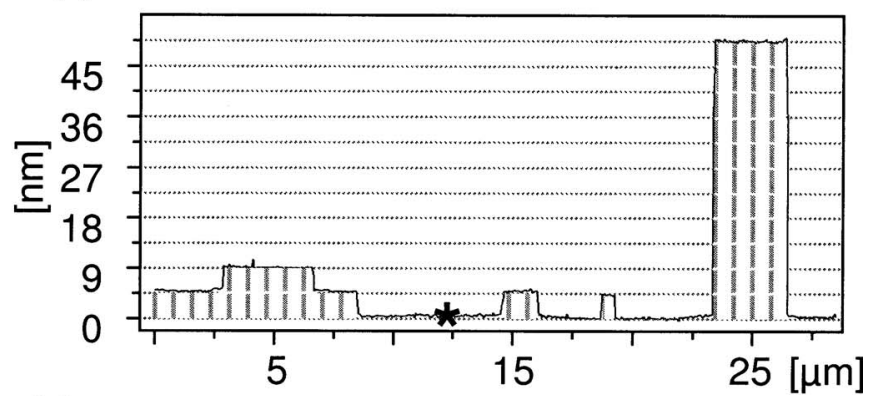

(d)

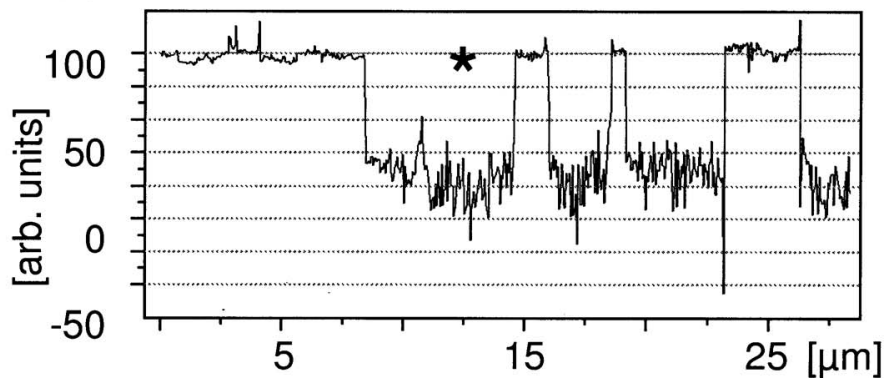

FIG. 1. (a) Topography and (b) friction images of a $25 \times 25 \mu \mathrm{m}^{2}$ area on a "thin" UV-cleaned sample (ellipsometric thickness of $64 \AA$ ). The friction image uses an inverted gray scale: light means low friction and darker areas correspond to higher friction. (c) A topographic cross section along the dashed line in (a) shows an island of a perpendicular layer partly covered with a second perpendicular layer before reaching the reference point (*); proceeding along a nearly perpendicular direction, we see two islands of a single perpendicular layer and then a bulk particle. All of these features have the same level of friction as shown in the cross section of the friction image in (d). In (c), symbols representing molecules illustrate how the step-height features can be fitted to an integer multiple of the all-trans length of the C32 molecule.

To achieve stable contact of the cantilever tip with the surface, we let it approach the film repeatedly until we observed a continuous and monotonic height signal after "snap in." In this height range, the normal force is increasing on approach due to the repulsive interaction with the film. We then shifted the set point of the feedback loop so that we operated in an attractive mode, with the magnitude of the normal force reduced below its value in the repulsive height range, in order to protect the film while scanning. The spatial resolution of the instrument normal to the surface was calibrated using a silicon grating (TGZ01 from MikroMasch). We estimate a noise-limited height resolution of $\sim 0.2 \mathrm{~nm}$ under conditions where the tip shape can be neglected and a lateral resolution of $\sim 0.1 \mu \mathrm{m}$ for $25 \times 25 \mu \mathrm{m}^{2}$ and $\sim 20 \mathrm{~nm}$ for $5 \times 5 \mu \mathrm{m}^{2}$ scans, respectively.

All of our AFM measurements were performed on the dip-coated samples at room temperature without thermal cycling of the sample above the bulk C32 melting point. We investigated a total of seven different samples and report on three representative samples here. On each of these samples, we investigated at least three different areas and recorded at least five scans on each. In all, we conducted over 800 scans on the three samples covering a total area of $\sim 5.6 \mathrm{~mm}^{2}$. Usually these scans began with an overview (i.e., a scan area of $100 \times 100$ or $\left.50 \times 50 \mu \mathrm{m}^{2}\right)$ and then zoomed into smaller areas $\left(<5 \times 5 \mu \mathrm{m}^{2}\right)$ for more detail. Successive scans allowed monitoring of the feedback signal stability and, more importantly, provided a check of whether scanning altered the film structure. With the exception of the "scraping" experiments described below, the structural features that we report survived successive scanning.

\section{RESULTS}

An AFM scan of a "thin" UV-cleaned sample $(\sim 64 \AA$ thick) is shown in Fig. 1. In the topography image [Fig. 1(a)], there are several large islands shaded in gray. These islands have a thickness of $\sim 4.3 \mathrm{~nm}$, which is approximately the all-trans length of the $\mathrm{C} 32$ molecule. Therefore, we tentatively interpret them as regions in which there is a single layer of molecules aligned with their long axis perpendicular to the surface. The two small spots circled in Fig. 1(a), one located at the top and the other at the bottom of the image, have a height corresponding to twice this thickness. A larger region of the same height (area $\approx 7.5 \mu \mathrm{m}^{2}$ ) is located at the bottom of the image. These islands are most easily interpreted as consisting of two layers of molecules oriented perpendicular to the surface. We will refer the dark areas in Fig. 1(a) between islands of perpendicular molecules as "ground level" and will later identify them with one to two layers of molecules oriented with their long axis parallel to the surface. Within the dark area is a bulk particle (white in color).

All of these topographic features appear in the height cross section taken along the dashed line in Fig. 1(a) consisting of two nearly perpendicular segments that meet at the point marked by the symbol (*). This cross section is shown in Fig. 1(c): Along the horizontal segment, we first see a region containing both a single- and a double-perpendicular layer before arriving at the point labeled $(*)$; then, along the nearly vertical segment in Fig. 1(a), we see two smaller regions with a single perpendicular layer and then a bulk particle of a height of $51 \pm 1 \mathrm{~nm}$. The particle height was determined from a height distribution measured in a smaller scan area centered on the particle. 


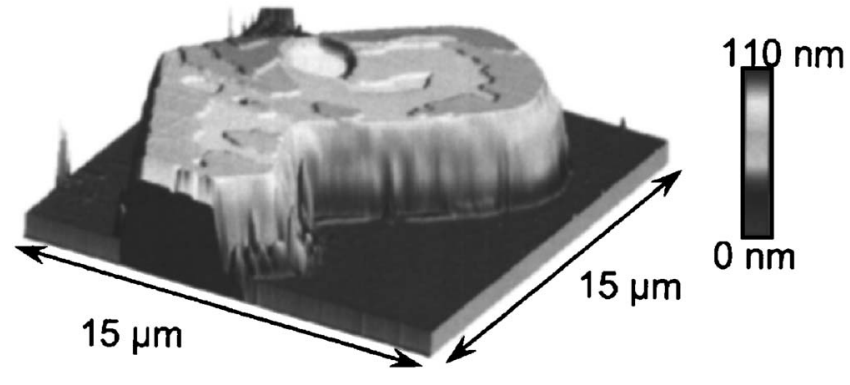

FIG. 2. A three-dimensional topographic image of a typical bulk particle from a "thick" UV-cleaned sample (ellipsometric thickness of $96 \AA$ ). The total height of this particle ranges from 110 to $140 \mathrm{~nm}$ due to its terrace structure. The terrace depth is comparable to the all-trans length of a C32 molecule. Note that the sides of the particle represent a convolution of the real topography and the shape of the pyramidal cantilever tip.

In the simultaneously recorded friction image in Fig. 1(b), there are only two major levels. The lower friction level (light gray) occurs on the surface of the perpendicular islands, regardless of the number of layers, as well as on top of the bulk particle. The higher friction level (dark gray) corresponds to the area that we have denoted ground level between the perpendicular islands. These two levels of friction are also apparent in the cross section shown in Fig. 1(d) taken along the same path as the height cross section in Fig. 1(c). Note that the steplike changes in friction coincide precisely with the step changes in topography.

Our observation that the top of the bulk particles has the same level of friction as the single- and double-perpendicular layers suggests that these particles consist of C32 molecules oriented with their long axis perpendicular to the substrate. In order to substantiate this interpretation, we focused on "thick" UV-cleaned samples (ellipsometric thickness of $96 \AA$ ) whose images tend to contain a larger number of bulk particles. An example of a bulk particle found on such a sample is shown in Fig. 2.

The bulk particle in Fig. 2 is a mesalike crystallite, ${ }^{5}$ with a total height of $110-140 \mathrm{~nm}$ and a terrace structure on its top. The step height from one terrace level to the next is about equal to the all-trans length of the molecule $(4.3 \mathrm{~nm})$ consistent with molecules oriented with their long axis perpendicular to the substrate. A simultaneously recorded friction image (not shown) confirms that, as for the thinner sample in Fig. 1(d), a region of high friction surrounds the bulk particle in Fig. 2 in contrast with the low-friction level found on its top.

In analyzing a subset of our scans, we found more than 300 bulk particles over a total scan area of nearly $1 \mathrm{~mm}^{2}$. Less than $10 \%$ of these particles were surrounded by the surface of a perpendicular layer (low friction) and these had either a small, cylindrically shaped footprint less than $5 \mu \mathrm{m}^{2}$ in area or had a small height $(<50 \mathrm{~nm})$. Bulk particles with a larger area and height were always found separated from perpendicular islands by a region having the higher level of friction.

The spatial extent of the perpendicular islands depends on the $\mathrm{C} 32$ coverage and the cleaning method. However, on no sample did we observe a perpendicular layer of molecules covering the entire scan area. The films prepared on an acidcleaned substrate showed larger areas of perpendicularly oriented molecules. The higher-coverage thick samples showed more islands with multiple perpendicular layers but the first perpendicular layer was never complete.

As an example of an acid-cleaned sample with large areas of perpendicular layers, we show AFM images of a thick film in Fig. 3. The topographic image in Fig. 3(a) shows an incomplete perpendicular layer (1) and a bulk particle (4) separated by a high-friction region which we again refer to as ground level (2), (3). The bulk particle has a height of $31.8 \pm 0.6 \mathrm{~nm}$ above the ground level. The first perpendicular layer has several holes (colored black) at locations A1, C1, and (5). There are also two regions of a double perpendicular layer (6, (7)). The step height from the ground level to the
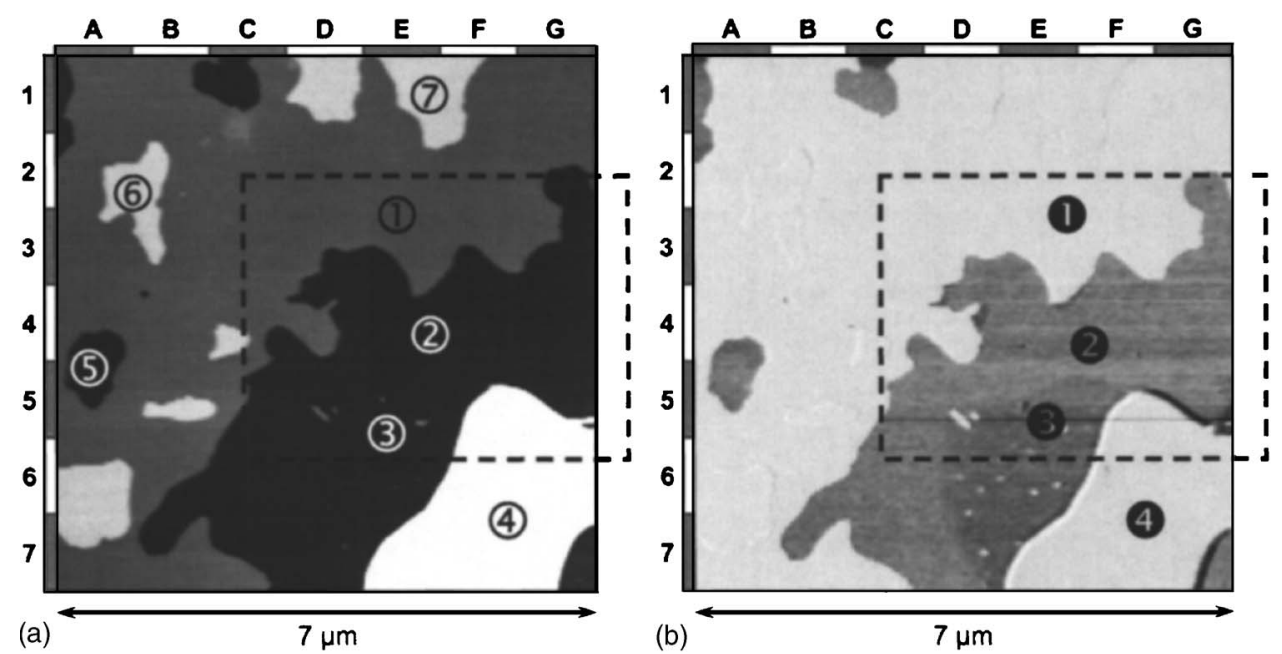

FIG. 3. (a) Topography and (b) friction images of a $7 \times 7 \mu \mathrm{m}^{2}$ area on a "thick" acid-cleaned sample (ellipsometric thickness of $94 \AA$ ). The linear gray scale in the topographic image (from black to white) covers a range of $15 \mathrm{~nm}$ [higher features such as the bulk particle (4) appear white]. The friction image uses an inverted gray scale: light and dark correspond to low and high friction, respectively. The topography image (a) shows a perpendicular island (1) with smaller areas of a second perpendicular layer (6), (7) as well as part of the large bulk particle (4) surrounded by "ground level" (2), (3)). The simultaneously recorded friction image (b) contains areas labeled with numbered filled circles. It shows only two major levels of friction: low $(\mathbf{1}, \mathbf{4})$ and high $(\mathbf{( 2 )}, \mathbf{3})$. The higher friction level splits in two sublevels as discussed in the text. The area in the outlined box is shown in more detail in Fig. 4. 

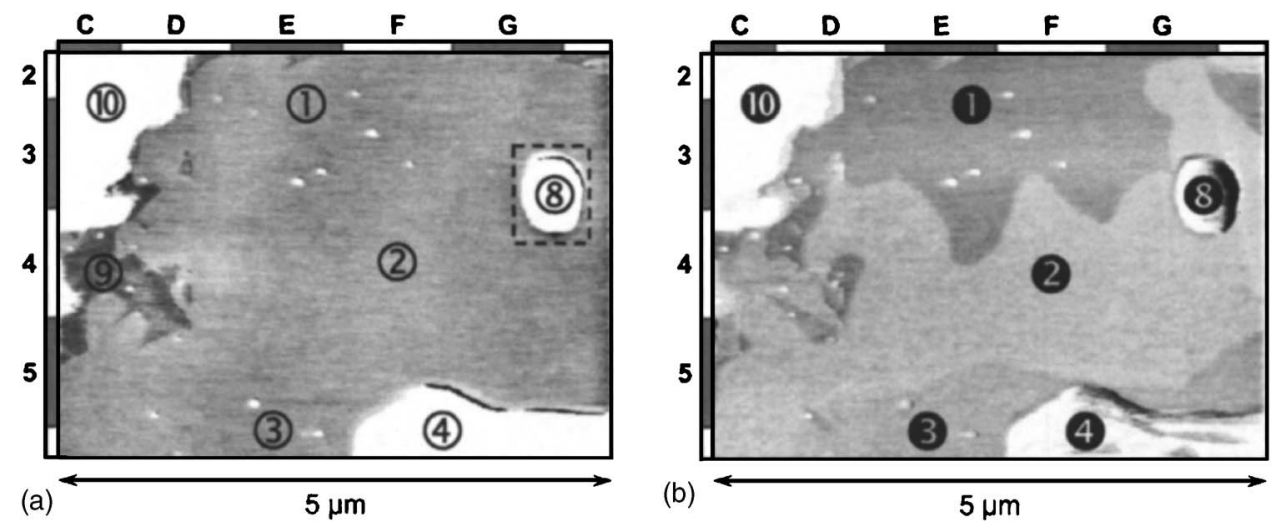

FIG. 4. (a) Topography and (b) friction images of a $5 \times 3.7 \mu \mathrm{m}^{2}$ area on the same "thick" acid-cleaned sample as in Fig. 3 after "scraping" (see text). The scan field is that outlined by the dashed box in Fig. 3. The linear gray scale in the topographic image (a) has a range of $3.5 \mathrm{~nm}$ (higher features appear white), while the inverted gray scale of the friction image (b) is comparable to that in Fig. 3(b). The topographic image in (a) shows that part of the bulk particle (4)) and a remnant of the perpendicular layer (10) are now separated by a region at "ground level" (1), (2), (3)). This ground-level region has different sublevels of friction with the area having the same shape as the perpendicular layer (1) in Fig. 3.

first perpendicular layer (from (2) to (1) is $4.4 \pm 0.2 \mathrm{~nm}$ as determined from the measured height histograms. The step heights from the first to the second perpendicular layers are also about equal to the all-trans length of the $\mathrm{C} 32$ molecule: height histograms give $4.2 \pm 0.2 \mathrm{~nm}$ for the steps (1)-(6) and $4.5 \pm 0.2 \mathrm{~nm}$ for the steps (1)-(7). The hole labeled (5) has a depth of $5.4 \pm 0.2 \mathrm{~nm}$ (1)-(5)).

As was the case for the thin film in Fig. 1(b), the thick film in Fig. 3(b) shows only two major levels of friction: a higher level shown as dark gray $(\mathbf{2}, \mathbf{3})$ and a lower level shown as light gray $(\mathbf{1}, \mathbf{4})$. Again, the single and double perpendicular layers as well as the top surface of the bulk particle all have the lower level of friction. Also, the groundlevel region and the bottoms of the holes in the first perpendicular layer have the higher friction level. Close inspection shows that within the darker-gray ground-level region, there are two sublevels of friction. There is a slightly darker level (3) adjacent to the bulk particle (4) and a lighter level (2) closer to the first perpendicular layer (1). The topography image [Fig. 3(a)] gives an rms roughness of $0.17 \mathrm{~nm}$ in the corresponding regions (2) and (3) without a distinct boundary between them as in the friction image [Fig. 3(b)].

After recording the images shown in Fig. 3, we performed scraping scans with an increased normal force in a small area around (1) and (2) with the aim of removing part of the perpendicular layer to reveal what was underneath. After successfully removing molecules from the perpendicular layer, we scanned a slightly larger field outlined by the dashed box in Fig. 3 using as small a normal force as possible. This larger field is shown in Fig. 4.

As a result of scraping, we can see in the topographic image of Fig. 4(a) that the area of the first perpendicular layer (10) has been reduced in size and the ground-level region between the bulk particle (4) and the first perpendicular layer has now expanded to include areas labeled (1), (2), and (3). In addition, new features labeled (8) and (9) appear. Area (9) is lower than the ground level with a step down of $0.7 \pm 0.15 \mathrm{~nm}$ that is considerably larger than the rms roughness $(0.17 \mathrm{~nm})$.

Figure 4(b) contains the friction image obtained in this scan area (dashed box in Fig. 3). We see that the groundlevel region is now split into three sublevels of friction $(\mathbf{1}$, (2, (3) even though we measure a constant height. It is interesting to note that the shape of the boundary between areas (1) and (2) in the friction image after scraping matches that between the first perpendicular layer (1) and ground level (2) in the topographic image of Fig. 3(a) before scraping. As will be discussed below, this result seems to suggest that the shape of the domains of different friction at ground level dictate where perpendicular layers will grow. In Fig. 4(b), region 3 and the bulk particle (4) appear to have the same shape as in the friction image of Fig. 3(b). As in Fig. 3(b), the remnant of the first perpendicular layer (DI) and the top of the bulk particle (4) have the same level of friction.

In Fig. 5, we show the height distribution of the new particle (8) calculated in the area enclosed by the dashed line in Fig. 4(a). The maximum height of this particle is $70 \pm 1 \mathrm{~nm}$; and the strong first peak in the distribution represents the data points at ground level surrounding this particle. A three-dimensional (3D) topographic image shown in the inset reveals discrete steps that manifest themselves as oscillations in the height distribution. In the right part of the graph, which represents the upper part of the new particle,

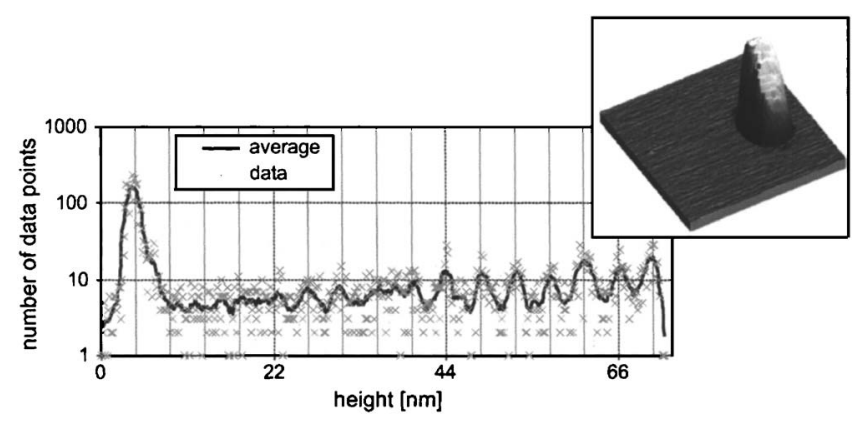

FIG. 5. Height distribution of the new particle made by scraping [labeled (8) in Fig. 4(a)]. The solid line represents a sliding average over seven consecutive data points (crosses). The total height of this particle is $70 \pm 1 \mathrm{~nm}$. The minor grid lines on the $x$ axis have a spacing of $4.4 \mathrm{~nm}$. The inset contains a $3 \mathrm{D}$ image of this particle showing discrete steps that manifest themselves as oscillations in the height distribution. 
the oscillation period is $\sim 4.4 \mathrm{~nm}$ as indicated by the minor gridlines, again consistent with terrace heights corresponding to the length of the C32 molecule.

\section{DISCUSSION}

In this section, we summarize the results of our AFM measurements concerning the stacking sequence and the spatial extent of the C32 layers as well as the structure, morphology, and location of bulk C32 particles on the $\mathrm{SiO}_{2}$ surface. We also discuss the observed relation between the topography and lateral friction levels.

\section{A. Evidence for parallel layers}

In our samples, the first layer above the natural oxide on the $\mathrm{Si}(100)$ surface consists of $\mathrm{C} 32$ molecules with their long axis oriented parallel to the $\mathrm{SiO}_{2}$ surface. Our evidence for this conclusion comes from the topographic images in Figs. 3(a) and 4(a) for our acid-cleaned sample, which had an initial ellipsometric thickness of $94 \AA$. In Fig. 3(a), there is a hole (5) with a depth of $5.4 \pm 0.2 \mathrm{~nm}$ relative to the height of the surrounding layer (1). Since the step down from layers (1) to (2) is only $4.4 \pm 0.2 \mathrm{~nm}$, this implies that the bottom of hole (5) is about $1 \mathrm{~nm}$ below the height of area (2).

Furthermore, since no deeper holes were observed, we interpret the bottom of hole (5) as the bare $\mathrm{SiO}_{2}$ surface. A $\sim 1 \mathrm{~nm}$ thickness for region (2) is consistent with one to two layers of $\mathrm{C} 32$ molecules oriented with their long axis parallel to the $\mathrm{SiO}_{2}$ surface, assuming a width of the $\mathrm{C} 32$ molecule of $\sim 0.5 \mathrm{~nm}$ as determined by x-ray specular reflectivity measurements on similarly prepared films ${ }^{10}$ and neutrondiffraction measurements of C32 monolayers adsorbed on graphite. ${ }^{15}$ Further evidence of at least one parallel layer is shown in Fig. 4(a) recorded after scraping this sample. In the area (9) we measured a step down of a depth of $0.7 \pm 0.15 \mathrm{~nm}$, which is also on the order of the width of a C32 molecule or the thickness of a parallel layer. Thus we conclude that the areas referred to as ground level in the topographic images of Figs. 3(a) and 4(a) correspond to the parallel layer(s) of C32 molecules. The height resolution of our measurement is insufficient to determine whether these areas are one or two parallel layers thick.

The fact that we observe very few holes in the parallel layers with which to determine their thickness suggests that these layers have a very large lateral extent, covering virtually the entire $\mathrm{SiO}_{2}$ surface. This contrasts with that of the perpendicular layers on the same samples that never completely cover a scan area.

In comparing the topographic and friction images of this sample, we have noted that areas (2) and (3) in Fig. 3(a) are both at ground level whereas the friction image in Fig. 3(b) indicates that these two areas have a different level of friction. We believe the most plausible explanation for different levels of lateral friction on the parallel layers without corresponding topographic changes is the presence of domains consisting of molecules that share a common azimuthal orientation. Crossing to an adjacent domain having a different azimuthal orientation would produce a step in friction. For example, one might expect that scanning the AFM tip in a direction parallel to the long axis of the molecule would produce a different lateral frictional force than perpendicular to it. Such an interpretation would lead us to conclude that the molecules in regions 2 and 3 in Fig. 4(b), for which the friction levels are close in magnitude, would have nearly the same azimuthal orientation compared with the molecules in the higher friction region $\mathbf{1}$.

A relation between lateral friction and azimuthal molecular orientation has also been suggested for polydiacetylene monolayers adsorbed on a mica substrate. ${ }^{2}$ In this case, a large friction anisotropy is observed scanning parallel and perpendicular to the molecular backbone, an effect attributed to anisotropic film stiffness. Molecular-dynamics simulations of tetracosane (C24) monolayers on a graphite surface offer some evidence of in-plane elastic anisotropy. ${ }^{16}$ They show a different rate of the molecule's oscillatory center-of-mass motion depending on whether the motion is along the direction of the $\mathrm{C} 24$ chains or perpendicular to it.

\section{B. Evidence for perpendicular layers}

We next summarize our evidence for layers of molecules oriented perpendicular to the surface and situated above the parallel layers. In Sec. III, we presented topographic images from both the 64- and 94- $\AA$-thick samples (Figs. 1 and 3, respectively) that consistently showed regions with step heights above ground level close to the all-trans length of the C32 molecule $(\sim 43 \AA) .{ }^{10}$ In addition, we found smaller regions that showed a second step upward of about this magnitude. Our friction measurements are consistent with this identification of perpendicular layers in that we measured the same magnitude of the lateral friction force on the top of both single and double layers.

All of the friction images are consistent with the parallel layer(s) lying underneath the perpendicular layer(s). For example [in Fig. 3(b)], we identified the lower-height regions (2) and 3 as a parallel layer with a high level of friction whereas the higher regions (1) and 4 had a lower level of friction by a factor of $2.5 \pm 0.5$. This difference in the lateral frictional force cannot be explained by a difference in the normal force. From force calibration curves, we found less than a $10 \%$ variation in the normal force on moving from a parallel to a perpendicular layer. Therefore, we attribute the lower level of friction in regions (1) and 4 [Fig. 3(b)] to the perpendicular orientation of the C32 molecules as inferred initially from the measured step height of these regions above ground level. We conclude that the molecular orientation is the dominant factor in determining the magnitude of the lateral frictional force.

Despite this apparent sensitivity of lateral friction to molecular orientation, we have not yet been able to resolve a difference between the magnitude of the frictional force on the bare $\mathrm{SiO}_{2}$ surface and on a layer of $\mathrm{C} 32$ molecules oriented parallel to the surface.

\section{Bulk particle structure and location}

The correlation between lateral friction and molecular orientation can also be used to elucidate the structure of bulk particles. As can be seen in Fig. 1(d), the top of the bulk 
particle shows the same friction level as the perpendicular layers which suggests that it consists of perpendicularly oriented molecules. This conclusion is consistent with the total height of this particle $(51 \pm 1 \mathrm{~nm})$ which is close to an integer multiple of the all-trans length of the C32 molecule (12 $\times 4.3 \mathrm{~nm}$ ). Further evidence supporting a perpendicular orientation of molecules within bulk particles is provided by the terrace structure on the particle in Fig. 2. In this case, the step heights between terrace levels are again close to the length of the fully extended C32 molecule. In making these comparisons between step heights and molecular length, we should note that the height resolution in our AFM measurements is insufficient to exclude tilting of the molecule away from the perpendicular orientation by an angle as large as $\sim 10^{\circ}$. However, a perpendicular orientation of the molecules is consistent with bulk particles having an orthorhombic structure oriented with their $c$ axis normal to the $\mathrm{SiO}_{2}$ surface as inferred from x-ray scattering measurements on films similarly grown from solution. ${ }^{10}$

Like the molecules in the bulk particles of as-dip-coated samples, those in the particle created during scraping are also oriented perpendicular to the substrate. Region 8 of Fig. 4(a) is a small bulk particle that nucleated from molecules that were intentionally scraped away from the neighboring perpendicular layer. This particle also has a terrace structure with a $4.4 \mathrm{~nm}$ spacing between levels consistent with perpendicularly oriented molecules. However, both the 3D topographic image of this artificially grown bulk particle and its height distribution (Fig. 5) show that it does not have a mesalike shape as was the case for the particle in the as-dipcoated sample in Fig. 2. A flat-topped particle would have a second large peak in its height distribution. It is interesting that this bulk particle could be fabricated by manipulating the AFM tip at room temperature in the absence of a solvent. We have also grown similarly oriented orthorhombic particles without a solvent in synchrotron x-ray scattering experiments in which C32 films were vapor deposited onto a $\mathrm{Ag}(111)$ surface in ultrahigh vacuum. ${ }^{17}$

Our survey of a large number of bulk particles shows that, above a minimum size, they are surrounded by an area of high friction that we have identified with a layer of parallel-oriented molecules. Furthermore, our scraping experiments suggest that these parallel layers extend underneath the bulk particles.

\section{Composite model}

Figure 6 is a 3D topographic image illustrating the principal features of our model for the structure and growth of $\mathrm{C} 32$ on $\mathrm{SiO}_{2}$. It was taken on the same 94 - $\AA$-thick acidcleaned sample as in Figs. 3-5. We see that the substrate is covered by a nearly complete parallel layer (1) that may contain domains differing in the azimuthal orientation of the molecules (not shown). On top of this parallel film are regions that consist of either a single layer of perpendicular molecules (2) or smaller islands of double perpendicular layers. Other scan areas of this sample showed small islands

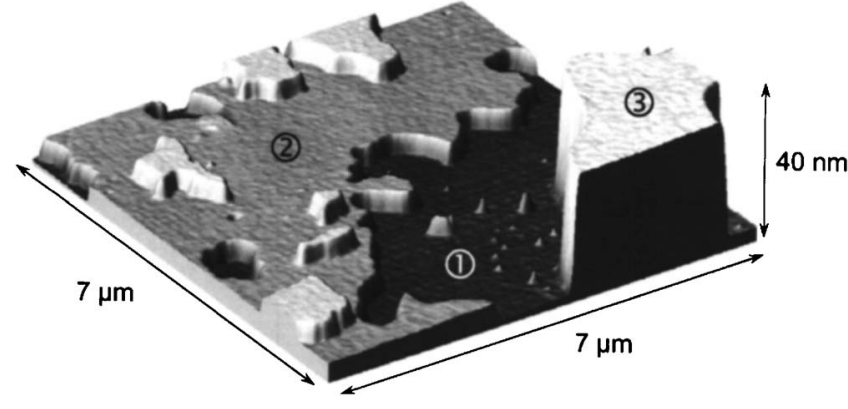

FIG. 6. A 3D topographic image of the acid-cleaned sample (ellipsometric thickness $\sim 94 \AA$ ) that contains the principal structural features of the C32 film. The dark areas (1) correspond to a parallel layer of C32 molecules that separates an incomplete perpendicular layer (2) from the bulk particle (3) in the right corner. At the left side of the image, on top of the perpendicular layer (2), there are several islands of a second perpendicular layer shown in midgray.

of triple or multiple perpendicular layers. In all cases, an area depleted of perpendicular molecules (1) surrounds the larger bulk particles (3).

\section{E. Comparison with previous AFM measurements}

Our results differ in some respects from previous AFM experiments conducted on C30 films spin coated onto $\mathrm{SiO}_{2} \cdot{ }^{6,7}$ We have not observed the fractal-like structure reported at submonolayer coverage in Ref. 6 apparently due to the higher coverage of our samples. However, the terraced islands reported by these authors at "excess coverage" are qualitatively similar to those in our samples shown in Figs. 2 and 5. Also, we have concluded that the larger bulk particles are more likely to reside on a layer of parallel-oriented molecules rather than on a perpendicular layer as described in Ref. 5. The area depleted of perpendicular molecules surrounding the larger bulk particles appears to be inconsistent with the closed alkane film described in Ref. 5.

\section{CONCLUSION}

The topographic model for C32 films deposited from solution on $\mathrm{a} \mathrm{SiO}_{2}$ surface that we have developed here from AFM measurements supports the novel growth mode that we inferred previously from ellipsometry and $\mathrm{x}$-ray experiments. ${ }^{9,10}$ We have confirmed the stacking sequence of one to two parallel layers of molecules adjacent to the $\mathrm{SiO}_{2}$ surface followed by perpendicular layers. Also, the step heights that we measured are consistent with the layer thicknesses determined from fitting $x$-ray specular reflectivity scans.

Our AFM results extend the ellipsometric and x-ray measurements in several respects. We find that the parallel layers cover virtually the entire scan areas so that their spatial extent is much greater than that of the perpendicular layers. The completeness of the parallel layers suggests that they are a thermodynamically stable phase that forms while the Si wafer is still immersed in the C32/heptane solution as was found many years ago for C32 films adsorbed on graphitized carbon substrates. ${ }^{18}$ Synchrotron x-ray scattering experiments are in progress on wafers immersed in a C32/ heptane solution in order to test this hypothesis. ${ }^{19}$ The 
sublevels in the lateral frictional force measured on the parallel layers suggest that they may consist of equal height domains in which the molecules share a common azimuthal orientation. We have proposed that the boundaries of these domains may dictate the size and shape of the perpendicular regions above them.

The models of the x-ray specular reflectivity curves ${ }^{10}$ measured on similarly prepared samples had suggested an incomplete perpendicular monolayer as based on its lower electron density compared with the parallel film layers; but the evidence was not nearly as direct as from our topographic and friction AFM images. Also, modeling of the X-ray specular reflectivity scans required only one perpendicular layer whereas our AFM scans have shown islands of multiple perpendicular layers that decrease progressively in area with their height above the parallel layers.

The origin of the partial layers of perpendicular molecules that form above the parallel layers remains uncertain. Their weak binding to the underlying parallel layers suggests that they are unlikely to adsorb while the wafer is immersed in solution. We suggest that they may form from residual droplets of solution after withdrawing the wafer. AFM measurements at higher temperatures are underway to investigate the stability of these partial perpendicular layers.

The submicron lateral resolution of AFM has allowed us to address the question of where the bulk particles in our samples are located. We observe the larger particles to be surrounded by regions of exposed parallel layers that most likely extend underneath the particles. Therefore, we consider it more probable that the bulk particles reside on a parallel layer rather than a perpendicular layer as had been inferred from our ellipsometry and x-ray measurements. ${ }^{9,10}$

The correlation that we have found between lateral friction and topography may not only be useful for resolving uncertainties in film structure but may also give an insight into the nanoscale lubricating properties of thin alkane films. For example, the dependence of lateral friction on molecular orientation that we have observed raises the question of whether the lubricating properties of alkane films can be improved by fabricating coatings in which alkane molecules are aligned perpendicular to a solid surface.

\section{ACKNOWLEDGMENTS}

We thank M. Grandbois and W.-C. Shin for fruitful discussions and preliminary measurements. We are grateful to $\mathrm{S}$. Magonov for sharing his AFM measurements on alkane films and for discussion of our results. We also thank $\mathrm{K}$. Knorr for a critical reading of the manuscript and many helpful discussions. This work was supported by U.S. National Science Foundation under Grant Nos. DMR-0109057 and DMR-0411748, by the Chilean government under FONDECYT Grant Nos. 1010548 and 7010548, and by the Fundacion Andes Grant No. C-13768.

${ }^{1}$ R. W. Carpick and M. Salmeron, Chem. Rev. (Washington, D.C.) 97, 1163 (1997).

${ }^{2}$ R. W. Carpick, D. Y. Sasaki, and A. R. Burns, Tribol. Lett. 7, 79 (1999).

${ }^{3}$ M. S. Marcus, R. W. Carpick, D. Y. Sasaki, and M. A. Eriksson, Phys. Rev. Lett. 88, 226103 (2002).

${ }^{4}$ J. C. Earnshaw and C. J. Hughes, Phys. Rev. A 46, R4494 (1992); X. Z. Wu, E. B. Sirota, S. K. Sinha, B. M. Ocko, and M. Deutsch, Phys. Rev. Lett. 70, 958 (1993); and X. Z. Wu, B. M. Ocko, E. B. Sirota, S. K. Sinha, M. Deutsch, B. H. Cao, and M. W. Kim, Science 261, 1018 (1993).

${ }^{5}$ C. Merkl, T. Pfohl, and H. Riegler, Phys. Rev. Lett. 79, 4625 (1997).

${ }^{6}$ A. Holzwarth, S. Leporatti, and H. Riegler, Europhys. Lett. 52, 653 (2000).

${ }^{7}$ H. Schollmeyer, B. Ocko, and H. Riegler, Langmuir 18, 4351 (2002).

${ }^{8}$ H. Schollmeyer, B. Struth, and H. Riegler, Langmuir 19, 5042 (2003).

${ }^{9}$ U. G. Volkmann, M. Pino, L. A. Altamirano, H. Taub, and F. Y. Hansen, J. Chem. Phys. 116, 2107 (2002).

${ }^{10}$ H. Mo, H. Taub, U. G. Volkmann, M. Pino, S. N. Ehrlich, F. Y. Hansen, E. Lu, and P. Miceli, Chem. Phys. Lett. 377, 99 (2003).

${ }^{11}$ S. R. Craig, G. P. Hastle, K. J. Roberts, and J. N. Sherwood, J. Mater. Chem. 4, 977 (1994).

${ }^{12}$ I. M. Tidswell, B. M. Ocko, P. S. Pershan, S. R. Wasserman, G. M. Whitesides, and J. D. Axe, Phys. Rev. B 41, 1111 (1990).

${ }^{13} \mathrm{~S}$. Trogisch and S. Magonov (unpublished).

${ }^{14}$ L. Nony, R. Boisgrad, and J. P. Aime, J. Chem. Phys. 111, 1615 (1999).

${ }^{15}$ K. W. Herwig, B. Matthies, and H. Taub, Phys. Rev. Lett. 75, 3154 (1995).

${ }^{16}$ A. D. Enevoldsen, M.S. thesis, Technical University of Denmark, 2003.

${ }^{17}$ H. Mo, S. Trogisch, H. Taub, S. N. Ehrlich, U. G. Volkmann, F. Y. Hansen, and M. Pino, Phys. Status Solidi A 201, 2375 (2004).

${ }^{18}$ A. J. Groszek, Proc. R. Soc. London, Ser. A 324, 473 (1970).

${ }^{19}$ M. Bai, S. Trogisch, S. N. Ehrlich, D. Wermeille, and H. Taub (unpublished). 